\title{
Caracterização mineralógica de solos tropicais por sensoriamento remoto hiperespectral(1)
}

\begin{abstract}
Marco Antonio Pizarro(2), José Carlos Neves Epiphanio ${ }^{(3)}$ e Lênio Soares Galvão(3)
Resumo - Dados hiperespectrais coletados no Brasil pelo sensor AVIRIS (Airborne Visible/Infrared Imaging Spectrometer) foram utilizados para a caracterização espectral de uma típica cena agropastoril e para testar o uso da técnica Spectral Feature Fitting (SFF) na identificação de minerais argilosos na imagem. Utilizou-se um modelo linear de mistura espectral, usando como membros de referência a vegetação verde e seca, a água, e os solos Nitossolo Vermelho, Latossolo Vermelho e Neossolo Quartzarênico órtico. Na identificação dos minerais, foram selecionados espectros de referência da biblioteca espectral do JPL/NASA. Os espectros dos pixels e das referências foram normalizados pelo método do contínuo espectral, entre 2.100 e $2.330 \mathrm{~nm}$, e depois comparados quanto à similaridade com o uso da técnica SFF. A caulinita predomina na cena, cuja identificação remota é dependente do tipo de solo e das proporções dos componentes da cena no interior do pixels. Os melhores resultados foram obtidos em solos de reflectância intermediária a alta e em pixels com valor de abundância da fração solo superior a 70\%. Isto ocorreu devido, respectivamente, à menor quantidade de substâncias opacas nestes solos e à redução nos pixels dos efeitos espectrais da lignina-celulose. Estes fatores tendem a mascarar as bandas de absorção das argilas.
\end{abstract}

Termos para indexação: sensores, reflectância, espectrometria, recursos minerais, identificação.

\section{Mineralogical characterization of tropical soils by hyperspectral remote sensing}

Abstract - AVIRIS (Airborne Visible/Infrared Imaging Spectrometer) data collected in Brazil were used for the spectral characterization of a typical crop-pasture scene, and for the evaluation of the Spectral Feature Fitting (SFF) technique for clay mineral identification in the image. A six-endmember linear spectral unmixing model was applied, consisting of green and senescent vegetation, water, and the soils Alfisol, Oxisol and Entisol. For mineral identification of kaolinite, montmorillonite and gibbsite in the AVIRIS image, reference spectra from the JPL/NASA spectral library were selected. Pixel and reference spectra were normalized by the continuum removal method, in the 2,100-2,330 nm interval, and then compared by the use of the SFF technique. Kaolinite is the dominant mineral in the scene, whose identification is dependent on the soil type and on the spectral mixture at sub-pixel level. The best results were obtained for soils with intermediate to high overall reflectance and for pixels with soil abundance values greater than $70 \%$, due, respectively, to the lower amount of opaque substances of these soils and to the reduction of spectral effects of the lignin-cellulose features. These factors tend to obliterate the absorption bands of clay minerals.

Index terms: sensors, reflectance, spectrometry, mineral resources, identification.

(1) Aceito para publicação em 19 de outubro de 2000.

(2) Instituto Nacional de Pesquisas Espaciais (Inpe), Divisão de Eletrônica Aeroespacial, Caixa Postal 515, CEP 12201-970 São José dos Campos, SP.

E-mail: pizarro@dea.inpe.br

(3) Inpe, Divisão de Sensoriamento Remoto.

E-mail: epiphani@1tid.inpe.br, lenio@ltid.inpe.br

\section{Introdução}

Recentes avanços na tecnologia de sensores remotos têm possibilitado a aquisição de dados de melhor resolução espectral do que os atualmente coletados por sensores multiespectrais mais tradicionais como, por exemplo, aqueles a bordo dos satéli- 
tes Landsat 7 e SPOT 4. Como exemplo, em dezembro de 1999, foi lançado pela NASA o satélite Terra do programa EOS (Earth Observing System). Esse satélite possui cinco instrumentos, dentre os quais o ASTER (Advanced Spaceborne Thermal Emission and Reflection Radiometer) com 14 bandas e o MODIS (Moderate Resolution Imaging Spectroradiometer) com 36 bandas, posicionadas nas faixas espectrais do visível e infravermelho próximo $(400-2.500 \mathrm{~nm}) \mathrm{e}$ infravermelho termal (8.000-15.000 nm). No final do ano 2000, foi lançado o satélite Earth Observing-1 com o sensor Hyperion (220 bandas), que constitui parte do programa de tecnologias revolucionárias proposto pela NASA para o terceiro milênio. Para o ano 2002, está planejado o lançamento do primeiro satélite comercial de alta resolução espectral, o ARIES (Australian Resource Information and Environment Satellite), com 105 bandas posicionadas no intervalo entre 400 e $2.500 \mathrm{~nm}$.

Essa nova fase de aquisição de dados tem recebido a denominação de Sensoriamento Remoto Hiperespectral ou Espectroscopia de Imageamento. De pixels representando a ocorrência de diferentes tipos de alvos na cena, pode-se extrair um espectro de reflectância diretamente da imagem em um nível de resolução espectral mais próximo do existente em condições de laboratório (Green et al., 1998). Quanto maior o número de bandas, melhor é a reconstituição de bandas de absorção espectral que podem ser usadas para identificação de alguns materiais na cena, incluindo a indicação, na imagem, da presença de minerais espectralmente dominantes nos espectros de solos. Embora a resposta espectral do solo seja influenciada por vários fatores que atuam em conjunto (matéria orgânica, óxidos de ferro, fração de argila, umidade), a reflectância de certos minerais, em alguns intervalos espectrais, se sobrepõe à do solo, o que possibilita a sua identificação remota através de dados de alta resolução espectral. Como exemplo, feições espectrais típicas do mineral caulinita em $2.200 \mathrm{~nm}$ podem ser também observadas nos espectros de laboratório de Alissolos, Latossolos e Cambissolos (Valeriano et al., 1995; Formaggio et al., 1996; Galvão et al., 1997; Demattê \& Focht, 1999).

Em geral, as técnicas de classificação usadas para identificação mineral através de sensoriamento remoto hiperespectral envolvem a comparação da similaridade dos espectros de reflectância extraídos diretamente das imagens com espectros de referência dos materiais testados para identificação na cena (Clark et al., 1990; Kruse et al., 1993). Entretanto, o uso dessas técnicas tem sido feito normalmente em regiões temperadas e em áreas com boa exposição de rochas e solos, o que favorece a ocorrência de bandas de absorção bem definidas nos espectros desses materiais, facilitando o processo de identificação mineral nas imagens hiperespectrais. De fato, pouco se conhece sobre os efeitos da intensa alteração mineral, típica dos ambientes tropicais, sobre esse processo, assim como sobre o impacto da mistura dos diferentes componentes da cena no interior do pixel.

O objetivo deste trabalho foi avaliar a utilização de dados hiperespectrais na caracterização espectral dos componentes de uma típica cena agropastoril e na identificação mineral remota em solos tropicais.

\section{Material e Métodos}

A área de estudo situa-se próximo à cidade de Campo Grande, MS, e às coordenadas $19^{\circ} 55^{\prime} \mathrm{S}$ e $53^{\circ} 30^{\prime} \mathrm{W}$. Os dados hiperespectrais foram coletados em agosto de 1995 pelo sensor AVIRIS (Airborne Visible/Infrared Imaging Spectrometer), operando a bordo de uma aeronave da NASA/JPL em 224 bandas (10 nm de largura) na faixa espectral de 400 a $2.500 \mathrm{~nm}$. A aquisição de dados fez parte de uma missão mais ampla: a SCAR-B (Smoke, Sulfate, Clouds And Radiation - Brazil). A área de estudo foi selecionada pela ausência de cobertura de nuvens e de fumaça na época da aquisição dos dados AVIRIS e por conter tipos distintos de solos com alguma variação mineralógica. Em um contexto regional, a área está situada na porção oeste da Bacia do Paraná, com topografia suave, vegetação natural de cerrado, extensas áreas de pastagem, e clima Tropical Brando de Transição com variação de temperatura média anual entre $19^{\circ} \mathrm{C}$ e $28^{\circ} \mathrm{C}$ (Fundação IBGE, 1989).

As imagens do sensor AVIRIS foram adquiridas na estação seca (agosto de 1995) em 224 bandas estreitas (10 nm de largura), posicionadas na faixa espectral de 400 a $2.500 \mathrm{~nm}$, com resolução espacial de 20 x $20 \mathrm{~m}$. Os dados, originalmente fornecidos em valores de radiância, foram convertidos para valores de reflectância de superfície, por um método de transferência radiativa baseado no modelo MODTRAN (Green, 1991). Em outras palavras, os efeitos de espalhamento e absorção atmosférica de gases como $\mathrm{H}_{2} \mathrm{O}, \mathrm{CO}_{2}, \mathrm{O}_{3}, \mathrm{O}_{2}$ e $\mathrm{CH}_{4}$ foram minimizados, o que constitui um pressuposto básico para possibilitar a compara- 
ção dos espectros de reflectância dos pixels com espectros de minerais de referência (bibliotecas espectrais) visando à sua identificação na cena.

Para caracterizar as variações espectrais associadas aos principais constituintes da cena e sua distribuição espacial, um modelo linear de mistura espectral (MLME) foi aplicado sobre os dados AVIRIS. O modelo assume que a reflectância de cada pixel é uma combinação linear da reflectância de membros de referência. Dessa forma, a abundância de cada membro de referência pode ser calculada para cada pixel (Adams et al., 1993). Uma descrição mais detalhada do modelo e um exemplo do cálculo da abundância dos membros de referência podem ser vistos em Roberts et al. (1998). No presente trabalho, optou-se pelo uso de um modelo com seis membros de referência (vegetação verde, vegetação seca, água, e três tipos de solos), definidos com base na escolha de pixels extremos em um diagrama de espalhamento resultante da aplicação de uma análise por componentes principais (ACP). Na estimativa da abundância, foi usado o método de decomposição do valor singular (Boardman, 1989). ACP é uma técnica comum usada para reduzir a dimensionalidade dos dados e identificar os fatores responsáveis pela variabilidade espectral (Smith et al., 1985; Galvão et al., 1997), mas também tem sido útil para seleção de membros de referência para modelos de mistura espectral (Bateson \& Curtiss, 1996). Os solos incluem Nitossolo Vermelho (NV), Latossolo Vermelho (LV) e Neossolo Quartzarênico órtico (RQo). A escolha da água em lugar da sombra deveu-se à dificuldade de identificar pixels de alvos sombreados em função das características planas do relevo. Em ambas as análises (ACP e MLME), as bandas ao redor dos intervalos de forte absorção atmosférica (1.400 e 1.900 nm) não foram consideradas. Mesmo após a correção atmosférica, esses dados não são úteis para a maior parte das aplicações envolvendo sensoriamento remoto, em função da forte opacidade da atmosfera nesses comprimentos de onda face à energia proveniente do Sol.

Finalmente, para fins de identificação mineral, aplicouse a técnica Spectral Feature Fitting (SFF). A técnica baseia-se na comparação, através do método dos mínimos quadrados, da similaridade de uma banda de absorção presente em um determinado intervalo espectral no espectro de um pixel com feições equivalentes presentes nos espectros de referências. Um exemplo do uso de uma técnica similar pode ser visto em Clark et al. (1990). As referências são definidas pelo usuário e podem incluir materiais como minerais, espécies de vegetação e alvos artificiais, de acordo com os objetivos da investigação. Esses espectros de referências constituem as denominadas bibliotecas espectrais, normalmente obtidas em laboratório ou campo, sob condições de aquisição de dados mais controladas.
Entretanto, antes da comparação propriamente dita, há necessidade de normalizar os espectros em ambos os conjuntos de dados (pixels e referência). O procedimento é ilustrado na Figura 1. Supondo que a banda de absorção em $2.200 \mathrm{~nm}$ seja a feição de interesse para identificação de minerais argilosos, ajusta-se uma função matemática, denominada contínuo linha-reta, nos limites da mesma. A normalização consiste em dividir o valor de reflectância do espectro original, em cada comprimento de onda, pelo valor equivalente projetado na função linha-reta. O objetivo é filtrar a banda de absorção dos espectros dos pixels e das referências, realçar a sua aparência e evitar pseudo-variações na posição de seu centro resultantes de mudanças na declividade dos espectros originais ou não-normalizados, que pode afetar a identificação mineral (Clark \& Roush, 1984). A comparação entre o espectro do pixel e o espectro de referência resulta em dois valores: um de escala e outro de erro médio quadrático (root mean square error RMSE). O primeiro está relacionado com a profundidade da banda de absorção sob análise, enquanto o segundo fornece uma medida relativa do erro de ajuste (mínimos quadrados) entre as feições do pixel e da referência. Pode-se então calcular uma razão escala/erro no processo de identificação mineral. Quanto maior for essa razão, maior será a probabilidade de um determinado pixel conter o material de referência testado quanto à presença na imagem.

No presente trabalho, a feição selecionada para a análise foi a banda de absorção centrada em $2.200 \mathrm{~nm}$, que está normalmente relacionada com processos de transições vibracionais produzidas pela interação da energia radiante com os minerais de argila (Hunt \& Salisbury, 1970). Os comprimentos de onda de 2.101 e $2.330 \mathrm{~nm}$ foram escolhidos como os limites da função contínuo linha-reta usada para a filtragem dessa banda de absorção dos espectros dos pixels e das referências. As referências incluíram os espectros dos minerais caulinita (PS-1A), montmorilonita (PS-2D) e gibbsita (OH-3A) (Figura 2), pertencentes à biblioteca espectral de domínio público do Jet Propulsion Laboratory (JPL/NASA). Esses minerais são de ocorrência comum nos solos tropicais brasileiros (Resende et al., 1988).

Finalmente, a imagem resultante da aplicação da técnica SFF, mostrando a distribuição de caulinita, montmorilonita e gibbsita na imagem AVIRIS, foi comparada com os resultados do modelo de mistura espectral, especialmente aos referentes à distribuição espacial dos três tipos de solo presentes na área de estudo. A análise de imagens foi feita usando o software ENVI (versão 3.2). Os resultados da identificação mineral remota também foram comparados com resultados de análises químicas e de difração de raios-X para amostras representativas desses solos. Os conteúdos de $\mathrm{SiO}_{2}, \mathrm{Al}_{2} \mathrm{O}_{3}, \mathrm{Fe}_{2} \mathrm{O}_{3}$ e $\mathrm{TiO}_{2}$ foram 

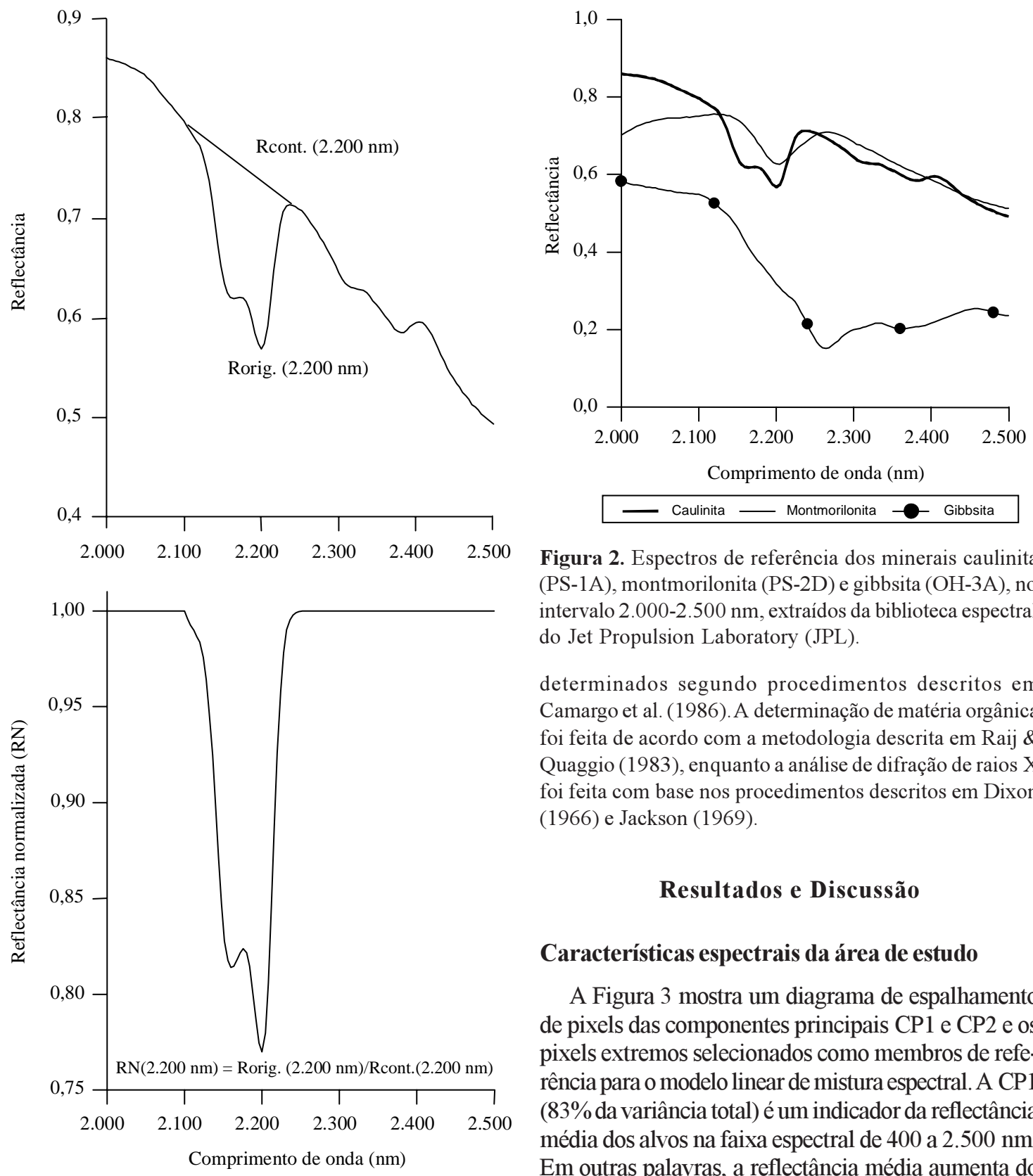

Figura 2. Espectros de referência dos minerais caulinita (PS-1A), montmorilonita (PS-2D) e gibbsita (OH-3A), no intervalo 2.000-2.500 nm, extraídos da biblioteca espectral do Jet Propulsion Laboratory (JPL).

determinados segundo procedimentos descritos em Camargo et al. (1986). A determinação de matéria orgânica foi feita de acordo com a metodologia descrita em Raij \& Quaggio (1983), enquanto a análise de difração de raios X foi feita com base nos procedimentos descritos em Dixon (1966) e Jackson (1969).

\section{Resultados e Discussão}

\section{Características espectrais da área de estudo}

A Figura 3 mostra um diagrama de espalhamento de pixels das componentes principais $\mathrm{CP} 1$ e $\mathrm{CP} 2 \mathrm{e}$ os pixels extremos selecionados como membros de referência para o modelo linear de mistura espectral. A CP1 (83\% da variância total) é um indicador da reflectância média dos alvos na faixa espectral de 400 a $2.500 \mathrm{~nm}$. Em outras palavras, a reflectância média aumenta do lado esquerdo do eixo CP1 na Figura 3 para o lado

Figura 1. Espectro de reflectância e de reflectância normalizada da caulinita, por meio da função contínuo (linha Rcont.) ajustada nos limites da banda de absorção. Um exemplo do cálculo envolvido na normalização é indicado para referência, em que Rorig. e Rcont. são, respectivamente, a reflectância em $2.200 \mathrm{~nm}$ do espectro original e o valor correspondente projetado na linha Rcont. direito, ou seja, de alvos como água e solos escuros do tipo NV para alvos como vegetação seca e solos claros do tipo RQo. Por outro lado, a CP2 (14\% da variância total) é útil para diferenciar vegetação verde (topo do eixo CP2 na Figura 3) dos outros componentes da cena. 
Os espectros de reflectância obtidos pelo sensor AVIRIS para os membros de referência indicados na Figura 3 são ilustrados na Figura 4. O espectro de vegetação verde é de mata de galeria, enquanto o de vegetação seca é provavelmente de uma cultura senescente de milho. O espectro da água é de um pequeno açude raso. Quando comparados com RQo, os solos LV e NV possuem menores valores de reflectância (menores escores CP1 na Figura 3) em razão de conterem maiores quantidades de matéria orgânica, ferro total $\left(\mathrm{Fe}_{2} \mathrm{O}_{3}\right), \mathrm{TiO}_{2}$ (Tabela 1) e minerais opacos. Estes constituintes tendem a reduzir a reflectância dos solos (Stoner \& Baumgardner, 1981; Henderson et al., 1992). No espectro de NV da Figura 4, uma fraca e ampla banda de absorção pode ser observada (indicada pela seta), a qual é produzida pela interação da energia radiante com óxidos de ferro (Townsend, 1987). No espectro de LV, a principal banda de absorção observada está centrada em torno de $2.200 \mathrm{~nm}$, sendo produzida pela interação da energia radiante com minerais argilosos (Hunt \& Salisbury, 1970; Hunt, 1980). Apesar de exibir uma menor quantidade de minerais argilosos, o espectro de RQo também exibe esta feição.

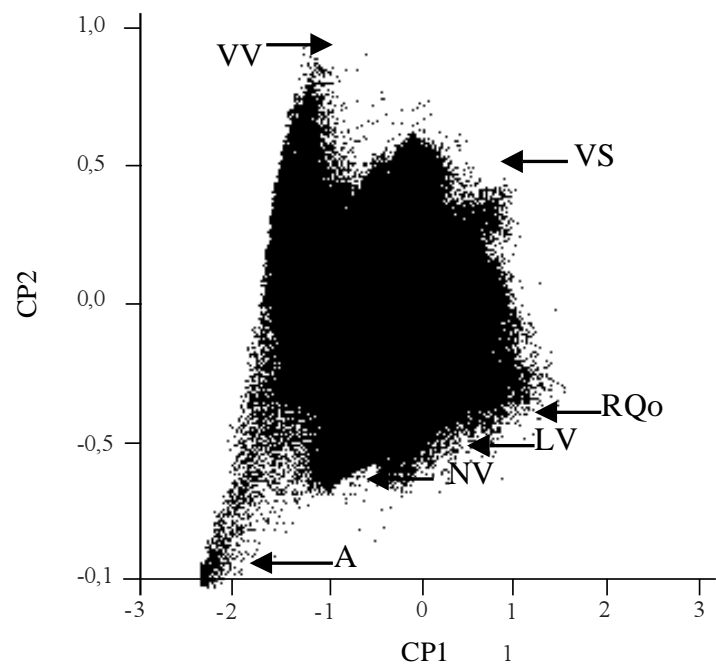

Figura 3. Diagrama de espalhamento dos pixels das componentes principais CP1 e CP2. A posição espectral dos membros de referência selecionados para o modelo de mistura espectral é mostrada: vegetação verde (VV), vegetação seca (VS), água (A), Nitossolo Vermelho (NV), Latossolo Vermelho (LV) e Neossolo Quartzarênico órtico (RQo).
A Figura 5a mostra os resultados de uma pósclassificação da imagem resultante da aplicação do modelo linear de mistura espectral. A Figura 5b ilustra uma composição colorida normal da área de estu-
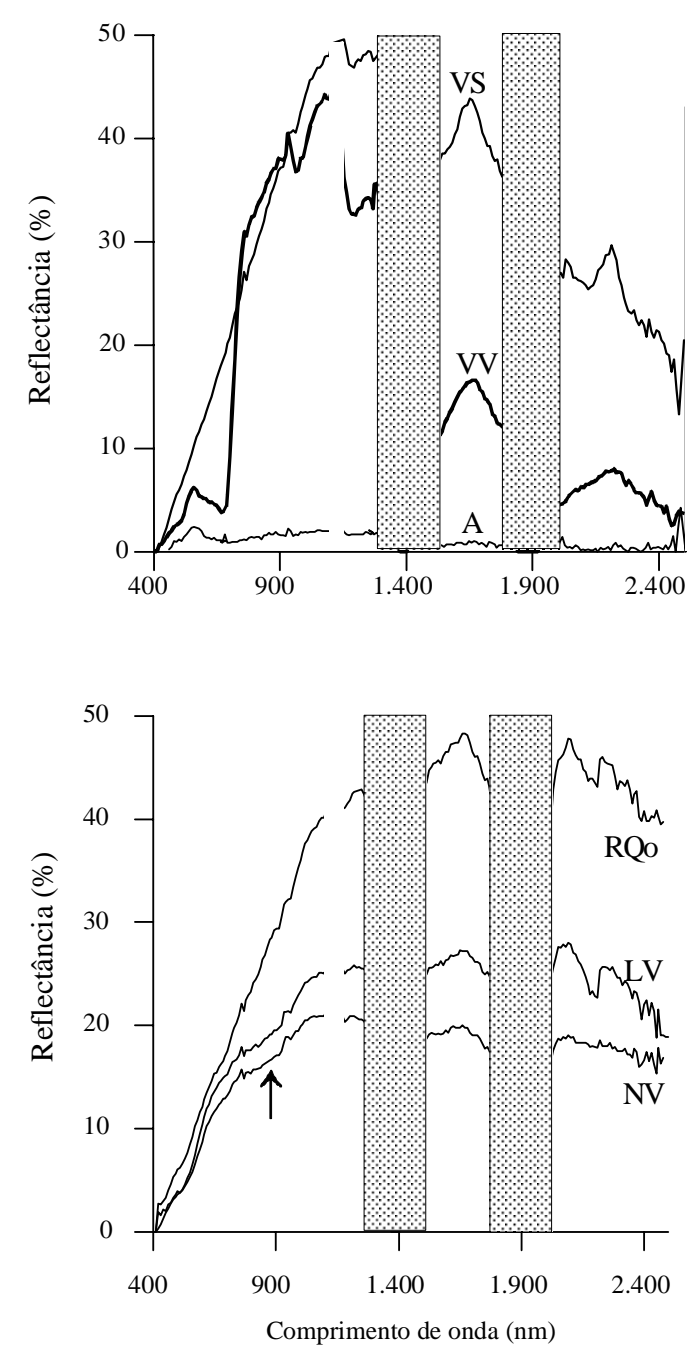

Figura 4. Espectros de reflectância AVIRIS dos membros de referência indicados na Figura 3. As colunas posicionadas próximas a $1.400 \mathrm{e} 1.900 \mathrm{~nm}$ indicam fortes intervalos de absorção atmosférica. Dados ruidosos em $1.140 \mathrm{~nm}$, resultantes do procedimento de correção atmosférica da banda de vapor d'água, foram omitidos para uma melhor representação gráfica. A seta indica a banda de absorção de óxido de ferro. 
do, obtida com o uso das bandas $31(677 \mathrm{~nm}), 19$ $(559 \mathrm{~nm})$ e $11(480 \mathrm{~nm})$ do sensor AVIRIS, respectivamente, em vermelho, verde e azul. Na Figura 5a, apenas pixels cujos membros de referência alcançaram valores de abundância superiores a $60 \%$ da somatória das frações foram realçados. Na Figura $5 b$, a localização aproximada dos pixels selecionados como membros de referência está indicada.

Conforme visto na Figura 5a, os pixels que contêm as maiores frações de vegetação verde estão associados com a mata ciliar ao longo do sistema de drenagem (áreas em ciano). A água, representada em preto na Figura 5a, está restrita a pequenas barragens ou a trechos do rio em que a mata ciliar não é tão densa, o que favorece o aparecimento da linha de drenagem. Em relação aos solos, os pixels mais puros de NV (vermelho na Figura 5a) ocorrem principalmente ao longo do Rio Pardo, enquanto os de LV (áreas em verde) são dominantes na porção centrosuperior da imagem. RQo (azul) é o solo dominante da área de estudo. Em geral, no entanto, a área de estudo é caracterizada espectralmente pela presença de vegetação seca (amarelo na Figura 5a), em razão da aquisição da imagem AVIRIS no mês de agosto, e por uma grande mistura dos diferentes componentes da cena (áreas em branco na Figura 5a). De fato, na composição colorida da Figura 5b, a vegetação verde ocorre de forma restrita às áreas de pastagem (verde claro) e às áreas de mata ciliar e de vegetação de cerrado (verde escuro). Os maiores valores de erro médio quadrático (RMSE) do modelo de mistura espectral estão associados à dificuldade de distinção espectral entre vegetação seca e RQo em algumas porções da área de estudo.

\section{Identificação mineral a partir das imagens}

A Figura 6 ilustra a distribuição dos minerais caulinita e montmorilonita ou dos pixels em que a banda de absorção, posicionada em seus espectros em torno de $2.200 \mathrm{~nm}$, reflete a presença deles. Estes minerais foram espectralmente identificados na imagem AVIRIS com o uso da técnica SFF, ou seja, através da comparação da similaridade entre os espectros dos pixels e os de referência (biblioteca espectral) destes minerais, ambos normalizados pelo método do contínuo espectral, conforme descrito anteriormente. Na realidade, os resultados apresentados na Figura 6 são de uma pós-classificação da imagem SFF, aplicada para realçar apenas pixels com valores de razão escala/erro superiores a 20, ou seja, pixels com maiores probabilidades de conter o mineral testado para identificação. Os resultados relativos à gibbsita não foram representados, porque este mineral não foi espectralmente identificado na imagem com base neste critério.

Observa-se que os pixels classificados da Figura 6 mostram estreita correspondência com as áreas de abundância de solos indicadas pelos resultados do modelo de mistura espectral (Figura 5a), especialmente nos solos LV e RQo. Dentre os minerais testados para classificação hiperespectral, a caulinita é o mineral espectralmente dominante na imagem e poucos são os pixels identificados como montmorilonita. Em relação aos demais minerais, a não-identificação espectral de gibbsita é consistente com os resultados de difração de raios-X mostrados na Tabela 1 , que indicam uma pequena porcentagem desse mineral nas amostras de solo da área de estudo.

Embora o NV seja o solo que contenha a maior fração de argila na Tabela 1, o pequeno número de

Tabela 1. Valores médios e desvio-padrão (entre parênteses) das análises químicas e mineralógicas de amostras dos solos Nitossolo Vermelho (NV), Latossolo Vermelho (LV) e Neossolo Quartzarênico órtico (RQo).

\begin{tabular}{|c|c|c|c|c|c|c|c|c|c|}
\hline Solo & $\mathrm{MO}$ & $\mathrm{SiO}_{2}$ & $\mathrm{Al}_{2} \mathrm{O}_{3}$ & $\mathrm{Fe}_{2} \mathrm{O}_{3}$ & $\mathrm{TiO}_{2}$ & Caulinita & Minerais $2: 1$ & Gibbsita & $\mathrm{N}^{(1)}$ \\
\hline & $(\mathrm{g} / \mathrm{kg})$ & & & & $(\%)$ & & & & \\
\hline NV & $\begin{array}{c}22,30 \\
(12,87)\end{array}$ & $\begin{array}{l}19,02 \\
(7,83)\end{array}$ & $\begin{array}{l}11,89 \\
(3,75)\end{array}$ & $\begin{array}{l}20,09 \\
(7,86)\end{array}$ & $\begin{array}{c}2,05 \\
(0,24)\end{array}$ & $\begin{array}{c}34,82 \\
(9,21)\end{array}$ & $\begin{array}{l}64,00 \\
(9,85)\end{array}$ & $\begin{array}{c}1,18 \\
(1,51)\end{array}$ & 10 \\
\hline LV & $\begin{array}{c}9,67 \\
(5,97)\end{array}$ & $\begin{array}{c}5,52 \\
(2,91)\end{array}$ & $\begin{array}{c}4,58 \\
(2,80)\end{array}$ & $\begin{array}{c}2,59 \\
(1,16)\end{array}$ & $\begin{array}{c}0,79 \\
(0,28)\end{array}$ & $\begin{array}{c}50,87 \\
(12,04)\end{array}$ & $\begin{array}{c}48,55 \\
(12,20)\end{array}$ & $\begin{array}{c}0,54 \\
(1,00)\end{array}$ & 12 \\
\hline RQo & $\begin{array}{l}10,00 \\
(3,16)\end{array}$ & $\begin{array}{c}3,61 \\
(1,24)\end{array}$ & $\begin{array}{c}2,34 \\
(1,43)\end{array}$ & $\begin{array}{c}1,00 \\
(0,36)\end{array}$ & $\begin{array}{c}0,44 \\
(0,08)\end{array}$ & $\begin{array}{l}56,43 \\
(6,38)\end{array}$ & $\begin{array}{c}43,57 \\
(6,38)\end{array}$ & $\begin{array}{c}0,00 \\
(0,00)\end{array}$ & 7 \\
\hline
\end{tabular}

${ }^{(1)} \mathrm{N}$ é o número de amostras usado nas determinações. 


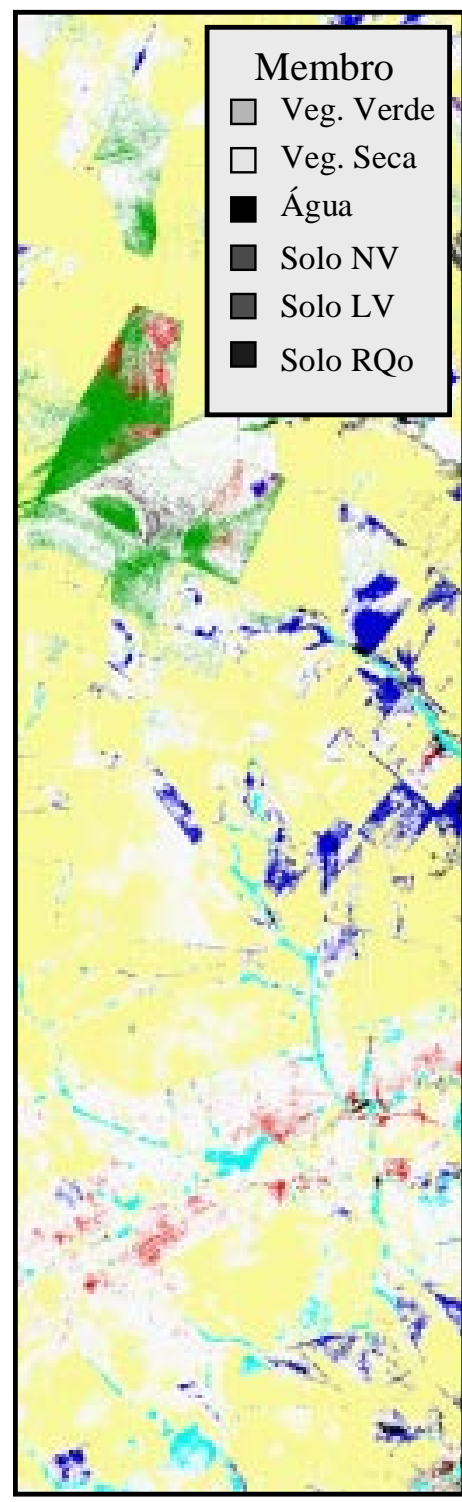

(a)

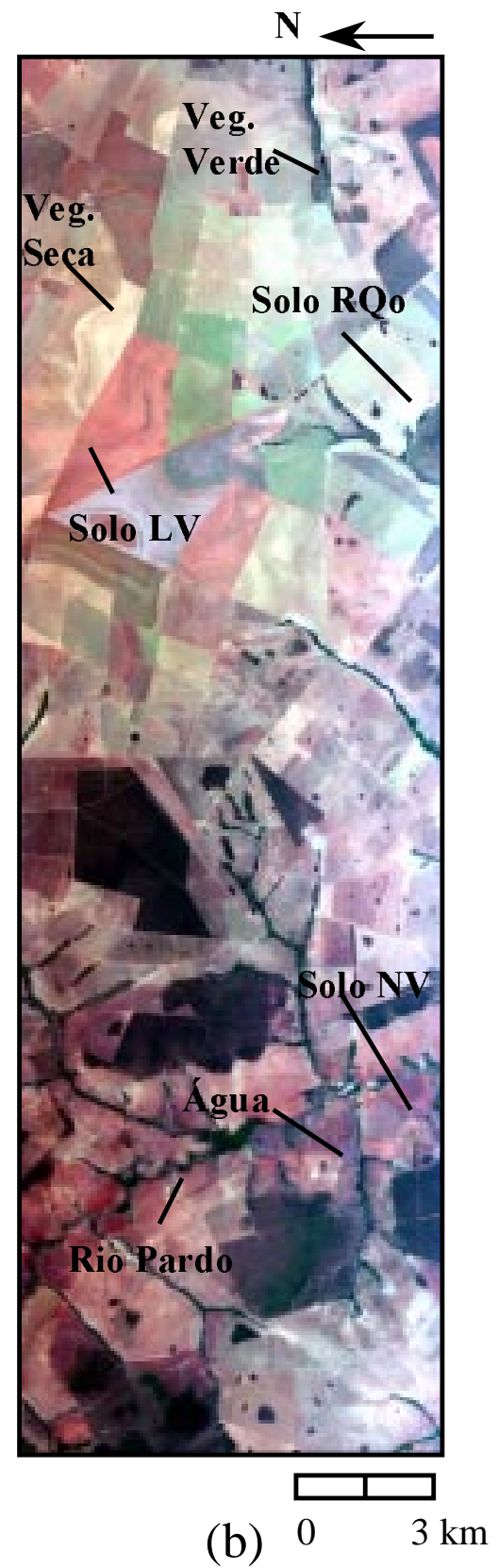

(b)

Figura 5. A imagem resultante do uso do modelo linear de mistura espectral, pósclassificada para realçar pixels com valores de abundância dos membros de referência superiores a $60 \%$ da fração total, é mostrada em (a). As áreas não-classificadas e, portanto, de intensa mistura espectral dos componentes da cena são indicadas em branco. Em (b), uma composição colorida normal é apresentada, obtida com as bandas $31(677 \mathrm{~nm}), 19(559 \mathrm{~nm})$ e $11(480 \mathrm{~nm})$ do sensor AVIRIS, respectivamente, em vermelho, verde e azul. A localização aproximada dos pixels selecionados como membros de referência é indicada. 
pixels classificados pela técnica SFF para este solo na Figura 6 deve-se a dois motivos. O primeiro está relacionado com a área de ocorrência deste tipo de solo ao longo do Rio Pardo, a qual é caracterizada na Figura 5a por uma grande mistura dos componentes de cena (áreas em cor branca). Conseqüentemente, há uma obliteração das bandas de absorção dos minerais de argila em $2.200 \mathrm{~nm}$ por feições de ligninacelulose resultantes da presença de vegetação verde ou seca, conforme discutido por Galvão et al. (1999). O segundo está relacionado com o fato de o NV possuir, em relação aos dois outros tipos de solos, uma maior quantidade de minerais opacos, conforme parcialmente indicado na Tabela 1 por maiores quantidades de ferro total e $\mathrm{TiO}_{2}$. As substâncias opacas não apenas reduzem a reflectância dos solos, como também afetam o surgimento de bandas de absorção bem definidas produzidas por outros constituintes (Stoner \& Baumgardner, 1981; Galvão \& Vitorello, 1998). Conseqüentemente, embora rico em minerais argilosos, o NV não mostra espectros com bandas de absorção de minerais argilosos bem definidas em $2.200 \mathrm{~nm}$, conforme ilustrado na Figura 4, o que dificulta a identificação destes minerais através de sensoriamento remoto hiperespectral.

De fato, a identificação mineral na Figura 6 ocorre principalmente para pixels com valores de abundância da fração solo superiores a 70\% na imagem do modelo de mistura espectral da Figura 5a. Isto explica a grande quantidade de pixels classificados nas áreas de domínio de LV, que são constituídas por solos expostos ou espectralmente mais puros, em relação aos demais, em função de sua preparação para a implementação de atividades agropastoris na época de aquisição da imagem AVIRIS.

É importante ressaltar que grande parte dos minerais passíveis de identificação mineral por sensoriamento hiperespectral em solos tropicais apresentam diferenças na posição dos centros de suas bandas de absorção. Como exemplo, na Figura 2, caulinita e montmorilonita mostram espectros com bandas de absorção centradas em torno de $2.200 \mathrm{~nm}$, enquanto o centro da banda no espectro de gibbsita está posicionado em $2.260 \mathrm{~nm}$. Outro exemplo inclui a distinção entre goetita e hematita que, em geral, mostram bandas de absorção centradas, respectivamente, em torno de 900 e $870 \mathrm{~nm}$ nos espectros de solos. Esse pequeno deslocamento na posição do centro das bandas pode ser um critério mais útil e

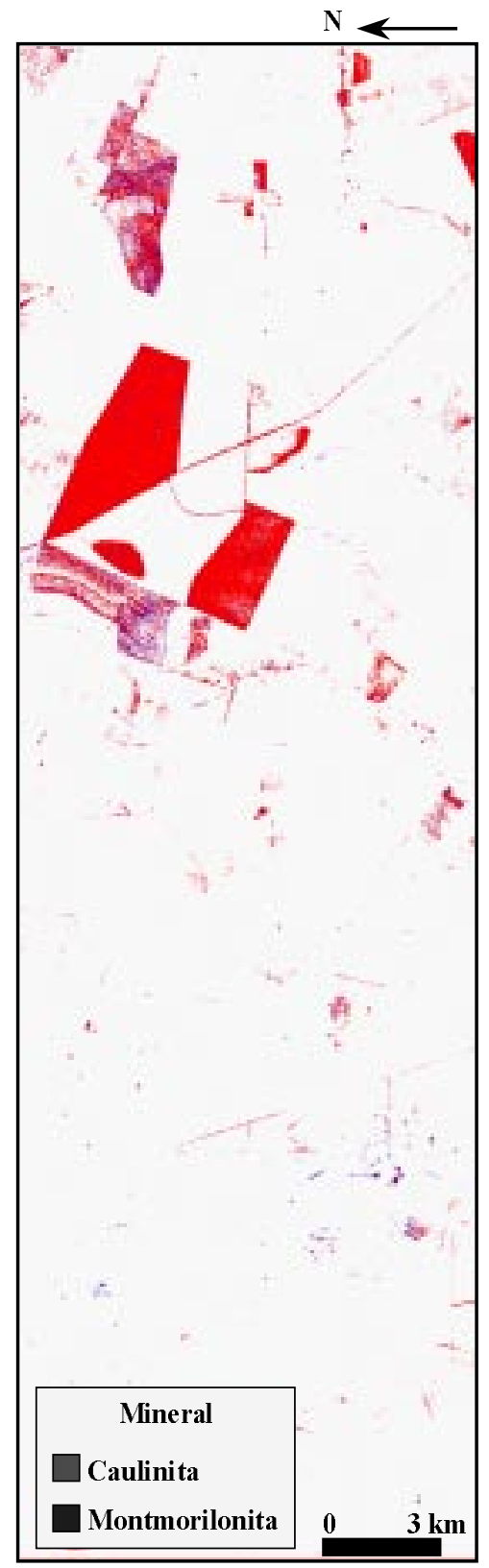

Figura 6. Identificação de áreas de domínio espectral dos minerais caulinita e montmorilonita na imagem AVIRIS, através do uso da técnica Spectral Feature Fitting (SFF). Os resultados mostrados são de uma pós-classificação da imagem SFF, aplicada para realçar pixels com valores de razão escala/erro superiores a 20. A gibbsita não foi identificada na imagem com base neste critério. As áreas não-classificadas são mostradas em branco. 
objetivo para a identificação destes minerais nas imagens hiperespectrais do que aquele resultante do uso do método dos mínimos quadrados, que forma a base conceitual da técnica SFF. De fato, resultados prévios do uso da técnica SFF para identificação desses óxidos na imagem AVIRIS da área de estudo mostram um domínio espectral acentuado de goetita que não é consistente com a presença de solos como $\mathrm{NV}$, que tendem a ter maiores quantidades de hematita (Pizarro, 1999).

Portanto, para otimizar o processo de identificação mineral em solos tropicais por sensoriamento remoto hiperespectral, há necessidade do uso de algoritmos que incluam critérios hierárquicos de inclusão/exclusão de minerais testados para a classificação. Um desses critérios de decisão é a comparação da posição do centro das bandas de absorção presentes nos espectros dos pixels com as existentes nos espectros dos minerais de referência (biblioteca espectral). Esse passo pode reduzir as possibilidades de identificação errônea de minerais nas imagens e anteceder a comparação propriamente dita da similaridade dos espectros com base no ajuste dos mínimos quadrados ou do uso da técnica SFF.

\section{Conclusões}

1. As imagens hiperespectrais auxiliam a delimitação de padrões tonais que estão relacionados com diferentes solos.

2. Os solos de maior albedo (LV e RQo) expressam mais nitidamente as feições de absorção que os solos de menor albedo.

3. A identificação mineral por meio de dados hiperespectrais é dependente do tipo de solo e da pureza espectral do pixel, e as melhores possibilidades de identificação estão associadas com solos de maior albedo ou com bandas de absorção mais bem definidas.

4. A classificação de áreas de ocorrência dos minerais montmorilonita, caulinita e gibbsita, a partir das imagens hiperespectrais, mostra boa relação com os tipos de solos em que esses minerais são esperados, desde que haja predomínio de solo exposto no pixel; é possível a geração de um mapa de distribuição espectral desses minerais.

5. A presença de vegetação verde ou seca no interior do pixel pode resultar em feições espectrais de lignina-celulose que tendem a mascarar as bandas de absorção das argilas, na faixa espectral de 2.100 a $2.300 \mathrm{~nm}$, e dificultar sua identificação mineral na cena.

6. Há necessidade de aprimoramento das técnicas de identificação mineral a partir de dados hiperespectrais.

\section{Agradecimentos}

Ao Laboratório de Análise de Solos do Departamento de Ciência do Solo da Escola Superior de Agricultura Luiz de Queiroz, pelas análises físico-químicas-mineralógicas das amostras de solos.

\section{Referências}

ADAMS, J. B.; SMITH, M. O.; GILLESPIE, A. R. Imaging spectroscopy: interpretation based on spectral mixture analysis. In: PIETERS, C. M.; ENGLERT, P. (Ed.). Remote geochemical analysis: elemental and mineralogical composition. New York: Cambridge University Press, 1993. p. 145-166.

BATESON, A.; CURTISS, B. A method for manual endmember selection and spectral unmixing. Remote Sensing of Environment, New York, v. 55, p. 229-243, 1996.

BOARDMAN, J. W. Inversion of imaging spectrometry data using singular value decomposition. In: INTERNATIONAL GEOSCIENCE AND REMOTE SENSING SYMPOSIUM, 1989, Vancouver. Proceedings... Ottawa: Institute of Electrical and Electronics Engineers, 1989. v. 4, p. 2069-2072.

CAMARGO, O. A.; MONIZ, A. C.; JORGE, J. A.; VALADARES, J. M. Métodos de análise química, mineralógica e física de solos do IAC. Campinas: Instituto Agronômico, 1986. 94 p. (Boletim Técnico, 106).

CLARK, R. N.; ROUSH, T. L. Reflectance spectroscopy: quantitative analysis techniques for remote sensing applications. Journal of Geophysical Research, Washington, v. 89, p. 6329-6340, 1984.

CLARK, R. N.; GALLAGHER, A. J.; SWAYZE, G. A. Material absorption band depth mapping of imaging spectrometer data using a complete band shape leastsquares fit with library reference spectra. In: AIRBORNE VISIBLE/INFRARED IMAGING SPECTROMETER (AVIRIS) WORKSHOP, 2., 1990, Pasadena. 
Proceedings... Pasadena: Jet Propulsion Laboratory, 1990 p. 176-186. (Publication, 90-54)

DEMATTÊ, J. A. M.; FOCHT, D. Detecção de solos erodidos pela avaliação de dados espectrais. Revista Brasileira de Ciência do Solo, Campinas, v. 23, p. 401413, 1999 .

DIXON, J. B. Quantitative analysis of kaolinite and gibbsite in soils by differential thermal and selective dissolution method. In: CLAYS AND CLAY MINERALS CONFERENCE, 14., 1966, Berkeley. Proceedings... New York: Pergamon, 1966. p. 82-90.

FORMAGGIO, A. R.; EPIPHANIO, J. C. N.; VALERIANO, M. M.; OLIVEIRA, J. B. Comportamento espectral (450-2450 nm) de solos tropicais de São Paulo. Revista Brasileira de Ciência do Solo, Campinas, v. 20 , p. $467-474,1996$

FUNDAÇÃO IBGE (Rio de Janeiro, RJ). Macrozoneamento geoambiental do Estado do Mato Grosso do Sul. Campo Grande: Seplan, 1989. 242 p.

GALVÃO, L. S.; VITORELLO, I. Role of organic matter in obliterating the effects of iron on spectral reflectance and color of Brazilian tropical soils. International Journal of Remote Sensing, London, v. 19, p. $1969-$ 1979, 1998.

GALVÃO, L. S.; VITORELLO, I.; ALMEIDA FILHO, R. Effects of band positioning and bandwidth on NDVI measurements of tropical savannas. Remote Sensing of Environment, New York, v. 67, p. 181-193, 1999.

GALVÃO, L. S.; VITORELLO, I; FORMAGGIO, A. R Relationships of spectral reflectance and color among nearand sub-surface horizons of tropical soil profiles. Remote Sensing of Environment, New York, v. 61, p. 24-33, 1997.

GREEN, R. O. Retrieval of reflectance from AVIRISmeasured radiance using a radioactive transfer code. In AIRBORNE VISIBLE/INFRARED IMAGING SPECTROMETER (AVIRIS) WORKSHOP, 3., 1991, Pasadena. Proceedings... Pasadena: Jet Propulsion Laboratory, 1991. p. 200-210. (Publication, 91-28)

GREEN, R. O.; EASTWOOD, M. L.; SARTURE, C. M. CHRIEN, T. G.; ARONSSON, M.; CHIPPENDALE, B. J.; FAUST, J. A.; PAVRI, B. E.; CHOVIT, C. J.; OLAH, M. R.; WILLIAMS, O. Imaging spectroscopy and the airborne visible/infrared imaging spectrometer (AVIRIS) Remote Sensing of Environment, New York, v. 65 , p. $227-248,1998$

HENDERSON, T. L.; BAUMGARDNER, M. F.; FRANZMEIER, D. P.; STOTT, D. E.; COSTER, D. C. High dimensional reflectance analysis of soil organic matter. Soil Science Society of America Journal, Madison, v. 56, p. $865-872,1992$
HUNT, G. R. Electromagnetic radiation: the communication link in remote sensing. In: SIEGAL, B. S.; GILLESPIE, A. R. (Ed.). Remote sensing in geology. New York: J. Wiley, 1980. p. 5-45.

HUNT, G. R.; SALISBURY, J. W. Visible and near-infrared spectra of minerals and rocks: I. silicate minerals. Modern Geology, London, v. 1, p. 283-300, 1970.

JACKSON, M. L. Soil chemical analysis: advanced course. Madison: Wisconsin University, 1969. 895 p

KRUSE, F. A.; LEFKOFF, A. B.; DIETZ, J. B. Expert system-based mineral mapping in Northern Death Valley, California/Nevada, using the airborne/infrared imaging spectrometer (AVIRIS). Remote Sensing of Environment, New York, v. 44, p. 309-336, 1993.

PIZARRO, M. A. Sensoriamento remoto hiperespectral para a caracterização e identificação mineral em solos tropicais. São José dos Campos: Inpe, 1999. 195 p. Dissertação de Mestrado.

RAIJ, B. van; QUAGGIO, J. A. Métodos de análise de solo para fins de fertilidade. Campinas: Instituto Agronômico, 1983. 40 p. (Boletim Técnico, 81).

RESENDE, M.; CURI, N.; SANTANA, D. P. Pedologia e fertilidade do solo: interações e aplicações. Brasília: Ministério da Educação, 1988. 83 p

ROBERTS, D. A.; BATISTA, G. T.; PEREIRA, J. L. G.; WALLER, E. K.; NELSON, B. W. Change identification using multitemporal spectral mixture analysis: applications in Eastern Amazonia. In: LUNETTA, R. S.; ELVIDGE, C. D. (Ed.). Remote sensing change detection environmental monitoring methods and applications. Chelsea: Ann Arbor, 1998. p. 137-161

SMITH, M. O.; JOHNSON, P. E.; ADAMS, J. B. Quantitative determination of mineral types and abundances from reflectance spectra using principal components analysis. Journal of Geophysical Research, Washington, v. 90, p. C797-C804, 1985

STONER, E. R.; BAUMGARDNER, M. F. Characteristic variations in reflectance of surface soils. Soil Science Society of America Journal, Madison, v. 45, p. 11611165,1981

TOWNSEND, T. E. Discrimination of iron alteration minerals in visible and near-infrared reflectance data. Journal of Geophysical Research, Washington, v. 92, p. 1441-1454, 1987

VALERIANO, M. M.; EPIPHANIO, J. C. N.; FORMAGGIO, A. R.; OLIVEIRA, J. B. Bi-directional reflectance factor of 14 soil classes from Brazil International Journal of Remote Sensing, London, v. 35, p. $113-128,1995$ 